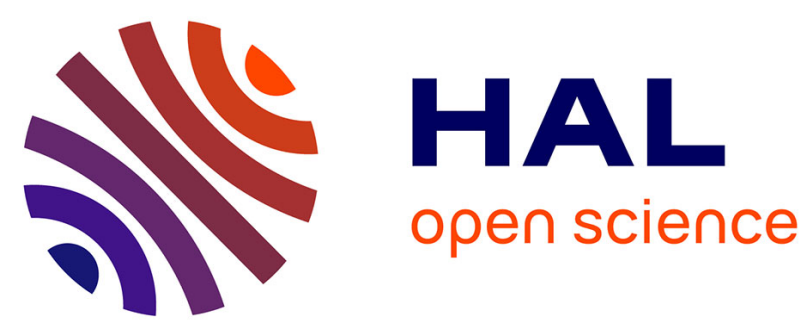

\title{
Épargne de précaution, réseaux sociaux et assurance chômage publique
}

Yann Algan, Pierre Cahuc, Bruno Decreuse, Francois Fontaine, Solenne

Tanguy

\section{- To cite this version:}

Yann Algan, Pierre Cahuc, Bruno Decreuse, Francois Fontaine, Solenne Tanguy. Épargne de précaution, réseaux sociaux et assurance chômage publique. Revue Française d'Economie, 2004, 19 (1), pp.3-36. 10.3406/rfeco.2004.1540 . hal-01038112

\section{HAL Id: hal-01038112}

https://hal-sciencespo.archives-ouvertes.fr/hal-01038112

Submitted on 23 Jul 2014

HAL is a multi-disciplinary open access archive for the deposit and dissemination of scientific research documents, whether they are published or not. The documents may come from teaching and research institutions in France or abroad, or from public or private research centers.
L'archive ouverte pluridisciplinaire HAL, est destinée au dépôt et à la diffusion de documents scientifiques de niveau recherche, publiés ou non, émanant des établissements d'enseignement et de recherche français ou étrangers, des laboratoires publics ou privés.

\section{(c)(1)}

Distributed under a Creative Commons Attribution| 4.0 International License 


\title{
Persée
}

http://www.persee.fr

\section{Epargne de précaution, réseaux sociaux et assurance chômage publique}

\author{
Pierre Cahuc;Yann Algan;Bruno Decreuse;François Fontaine;Solenne Tanguy \\ Revue française d'économie, Année 2004, Volume 19, Numéro 1 \\ p. 3 - 36
}

Voir l'article en ligne

Public Unemployment Benefits Versus Self-Insurance and Social Insurance This article reassesses the optimal level of public insurance schemes when pri- vate insurance with precautionary savings or familial insurance with social networks are taken into account. These features are found to deeply challenge the traditional efficiency and equity results attached to the provision of unemployment benefits and provide new room for public insurance.

\section{Avertissement}

L'éditeur du site «PERSEE » - le Ministère de la jeunesse, de l'éducation nationale et de la recherche, Direction de l'enseignement supérieur, Sous-direction des bibliothèques et de la documentation - détient la propriété intellectuelle et les droits d'exploitation. A ce titre il est titulaire des droits d'auteur et du droit sui generis du producteur de bases de données sur ce site conformément à la loi nº8-536 du 1er juillet 1998 relative aux bases de données.

Les oeuvres reproduites sur le site «PERSEE » sont protégées par les dispositions générales du Code de la propriété intellectuelle.

Droits et devoirs des utilisateurs

Pour un usage strictement privé, la simple reproduction du contenu de ce site est libre.

Pour un usage scientifique ou pédagogique, à des fins de recherches, d'enseignement ou de communication excluant toute exploitation commerciale, la reproduction et la communication au public du contenu de ce site sont autorisées, sous réserve que celles-ci servent d'illustration, ne soient pas substantielles et ne soient pas expressément limitées (plans ou photographies). La mention Le Ministère de la jeunesse, de l'éducation nationale et de la recherche, Direction de l'enseignement supérieur, Sous-direction des bibliothèques et de la documentation sur chaque reproduction tirée du site est obligatoire ainsi que le nom de la revue et- lorsqu'ils sont indiqués - le nom de l'auteur et la référence du document reproduit.

Toute autre reproduction ou communication au public, intégrale ou substantielle du contenu de ce site, par quelque procédé que ce soit, de l'éditeur original de l'oeuvre, de l'auteur et de ses ayants droit.

La reproduction et l'exploitation des photographies et des plans, y compris à des fins commerciales, doivent être autorisés par l'éditeur du site, Le Ministère de la jeunesse, de l'éducation nationale et de la recherche, Direction de l'enseignement supérieur, Sous-direction des bibliothèques et de la documentation (voir http://www.sup.adc.education.fr/bib/ ). La source et les crédits devront toujours être mentionnés. 


\section{Yann ALGAN \\ Pierre CAHUC}

Bruno DECREUSE

François FONTAINE

Solenne TANGUY

Epargne de précaution,

réseaux sociaux

et assurance chômage

publique

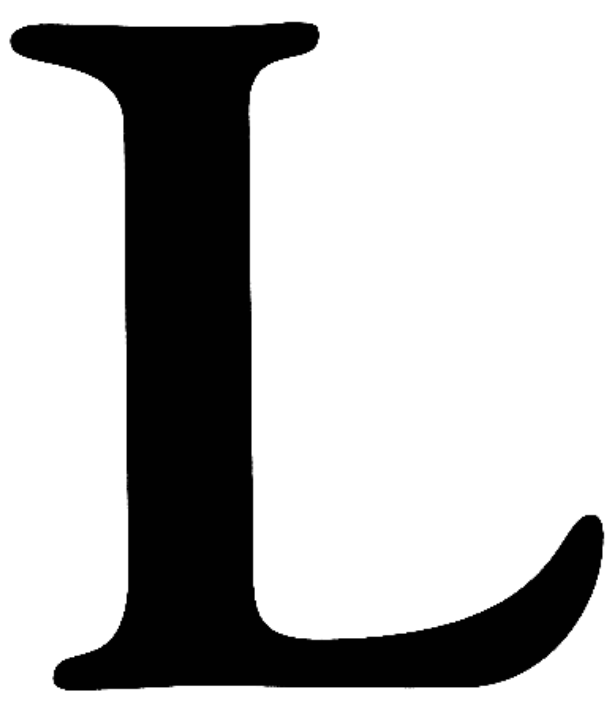

es systèmes publics d'assurance chômage ont pour vocation principale de prémunir les travailleurs 
contre de trop importantes fluctuations de leur consommation au cours des épisodes de chômage (Browning et Crossley [2001]) et de financer leur recherche d'emploi (Acemoglu et Shimer [1999]). L'intervention de l'autorité publique en ce domaine est habituellement justifiée par l'absence de systèmes privés d'assurance sur le marché du travail, résultant de la présence de sélection adverse et d'aléa moral spécifique à ce marché ( $c f$. Chiu et Karni [1998], Kiander [1993], Blanchard et Tirole [2003]). Cependant, l'efficacité de l'intervention publique en matière d'assurance chômage a été récemment mise en cause en arguant qu'il existe d'autres sources d'assurance à la disposition des travailleurs. Ceux-ci peuvent en effet s'auto-assurer, en constituant une épargne de précaution ${ }^{1}$ pendant les périodes d'emploi. Ils peuvent aussi bénéficier d'une assurance interpersonnelle, qui repose sur leur capital social, c'est-à-dire sur leurs réseaux familiaux et sociaux. La prise en compte de ces mécanismes complémentaires s'inscrit dans une réflexion contemporaine plus générale sur l'optimalité des systèmes d'assurance publique dans les pays de l'OCDE, comme en témoigne la récente réforme du PARE en France.

L'auto-assurance et l'assurance interpersonnelle peuvent-elles se substituer pour autant au système public d'assurance chômage ? Il est étonnant de constater que plusieurs travaux récents ont répondu par l'affirmative à cette question. Des travaux empiriques ont tout d'abord montré que les ménages considèrent de fait ces différentes formes d'assurance comme des proches substituts. Ainsi, selon l'étude de Engen et Gruber [2001] portant sur des ménages nord-américains, le comportement d'épargne des ménages les plus exposés au risque de chômage semble fortement conditionné par le niveau d'assurance publique dont ils peuvent disposer. Ces travaux mettent aussi en avant l'ampleur du niveau d'assurance que l'épargne et les réseaux familiaux peuvent potentiellement prodiguer. Ainsi Deaton [1991] et Aiyagari [1994] montrent-ils que l'épargne de précaution est susceptible d'offrir une assurance presque parfaite contre les risques individuels de revenus. Quant à l'assurance interpersonnelle, Bentolila et al. [2003] soulignent que 
les pertes de consommation provoquées par un épisode de chômage sont considérablement amoindries par la présence de réseaux sociaux. Dans ce contexte, il est peu surprenant que les études actuelles concluent au faible impact des systèmes publics d'assurance chômage lorsqu'il est tenu compte de ces deux mécanismes assurantiels supplémentaires. En particulier, les modèles qui réévaluent le niveau optimal des allocations chômage en tenant compte des comportements d'épargne, initiés par Hansen et Imrohoroglu [1992], concluent que le gain assurantiel des allocations chômage est nul (Wang et Williamson [2002]).

Dans cet article, nous soutenons que ce résultat provocateur repose sur des hypothèses simplificatrices, qui négligent, en particulier, l'hétérogénéité individuelle et donc le rôle joué par l'assurance chômage publique dans la réduction des inégalités. Cependant, nous soulignons aussi que le caractère excessif de ce résultat ne doit pas obérer la richesse d'une approche consistant à évaluer les conséquences des systèmes publics d'assurance chômage en tenant compte de leurs interactions avec d'autres sources d'assurance. Cette approche apporte en effet des éclairages novateurs sur l'efficacité de l'organisation de l'assurance chômage. Elle définit les droits et les devoirs des bénéficiaires d'allocations, en jouant notamment sur le montant et le profil temporel des allocations, sur les conditions d'éligibilité et les modalités de contrôle de l'activité de recherche d'emploi. Aujourd'hui, ce qui pose problème, ce n'est évidemment pas l'existence même des systèmes publics d'assurance chômage, mais bien la recherche d'une organisation efficace de ces systèmes. Or, l'efficacité d'une organisation ne peut être étudiée qu'en prenant en compte l'environnement dans lequel elle évolue. C'est à ce titre que les réflexions sur les interactions entre le système public d'assurance chômage, l'auto-assurance et l'assurance interpersonnelle sont riches d'enseignements. Nous montrerons, en analysant successivement l'influence de l'auto-assurance et de l'assurance interpersonnelle, qu'elles améliorent notre compréhension des conséquences du profil temporel de l'allocation chômage, du poids des normes sociales sur l'activité 
de recherche d'emploi et du rôle de coordination des choix des méthodes de prospection d'emploi joué par les systèmes publics d'assurance chômage.

\section{Assurance chômage et auto-assurance}

Les réflexions sur les bienfaits assurantiels des allocations chômage se sont tout d'abord enrichies par la prise en compte d'un mécanisme d'assurance individuelle supplémentaire : l'autoassurance sous forme d'épargne de précaution. Pour pallier l'absence actuelle de systèmes privés d'assurance contre les risques du marché du travail, un individu peut s'auto-assurer en se constituant un matelas d'épargne lorsqu'il est en emploi, dans lequel il pourra puiser pendant les périodes de chômage. Cette auto-assurance differe des mécanismes classiques d'assurance dans la mesure où elle opère une diversification des risques dans le temps au lieu d'une diversification entre individus pour une période donnée. Elle est, en ce sens, davantage tributaire de chaque histoire individuelle sur le marché du travail et peut accroître les inégalités entre individus dans la couverture des risques.

Dans quelle mesure ce comportement d'auto-assurance modifie-t-il les réflexions traditionnelles sur l'allocation chômage ? Nous allons répondre à cette question en trois étapes. La première consiste à comparer la capacité assurantielle de ces deux mécanismes face aux risques de chômage. La deuxième étape élargit le cadre traditionnel de la littérature des modèles de recherche d'emploi en évaluant également le rôle de l'épargne de précaution sur les stratégies de recherche d'emploi. Enfin la dernière étape logique consistera à s'interroger sur la façon dont l'épargne de précaution est susceptible de modifier les recom- 
mandations de politiques économiques sur le niveau optimal des allocations chômage.

\section{L'auto-assurance contre les risques de chômage}

En préalable à toute recommandation de politique économique, il semble nécessaire d'évaluer la capacité assurantielle de l'épargne de précaution par rapport au système d'assurance chômage. Cette réflexion se nourrit tout d'abord d'un constat empirique : il semblerait que les décisions d'épargne des ménages interagissent très fortement avec les mécanismes d'assurance publique. Engen et Gruber [2001] ont récemment montré sur un panel américain que les ménages sont d'autant moins enclins à s'auto-assurer contre les risques de chômage qu'ils peuvent compter sur des allocations chômage plus généreuses. Hubbard et al. [1995] observent le même type d'effet d'éviction de l'épargne par les mécanismes d'assurance publique dans le cas du Medicare (assurance publique de santé aux Etats-Unis). Au-delà des résultats quantitatifs, ces études empiriques suggèrent surtout que les ménages considèrent l'auto-assurance et l'assurance publique, en dépit de leur différence de nature, comme deux instruments substituables. Il semble donc essentiel de comparer plus en détail les capacités assurantielles de chacun d'entre eux.

Le second motif de comparaison est d'ordre théorique. De nombreux travaux ont évalué le coût en bien-être que représente l'absence de marchés complets d'assurance contre les risques individuels de revenu. L'incomplétude des marchés se caractérise par la présence d'un unique actif, identifié à l'épargne, pour se prémunir contre les différents aléas du marché du travail. Le résultat pourtant paradoxal de ces travaux est que l'épargne est susceptible d'offrir un lissage presque parfait de la consommation face aux variations de revenus. En particulier Aiyagari [1994] montre dans un modèle quantitatif avec des contraintes de liquidités que l'accumulation d'un actif certain permettrait à un individu de se constituer une assurance quasi complète contre les risques idiosyncrasiques de revenus. Dans ce contexte, l'assurance chômage 
aurait-elle encore un rôle à jouer si on lui substituait un système reposant uniquement sur l'épargne de précaution des ménages ? Algan et al. [2004] ont récemment contribué à répondre à cette question en comparant les capacités assurantielles du système public d'assurance chômage et du système d'auto-assurance par une épargne de précaution. Les agents se distinguent par deux sources d'hétérogénéité. La première est liée au marché du travail, les agents pouvant être dans deux états : soit employé, soit chômeur. La seconde est une hétérogénéité des richesses : les agents different dans leur niveau de stocks d'épargne en fonction de leurs trajectoires passées sur le marché du travail. Le modèle à agents hétérogènes est alors étalonné sur le marché du travail français caractérisé par un taux de chômage de $10 \%$ et une durée moyenne du chômage de 10 mois. Les auteurs proposent de comparer dans ce cadre les capacités assurantielles de l'épargne et des allocations chômage, en fixant ces dernières à $60 \%$ des revenus passés (Martin [1996]). Cette comparaison utilise l'indicateur du coût des fluctuations de revenu de Lucas [1987], qui mesure le pourcentage de consommation dont il faudrait dédommager un individu vivant dans une situation d'assurance parfaite (marchés complets $\mathrm{MC}$ ) pour qu'il soit indifférent à vivre dans une situation d'assurance incomplète, représentée ici par les économies avec épargne (marchés financiers MF) ou assurance chômage (AC).

Dans un premier temps les auteurs entreprennent ce calcul dans le cadre d'économies prises à l'état stationnaire, ce qui signifie qu'il n'est pas tenu compte de toute la transition d'accumulation du capital dans l'économie avec auto-assurance. Ce cadre est celui retenu actuellement par la littérature consacrée à la capacité assurantielle de l'épargne de précaution, notamment par Rogerson et Schindler [2002]. La première ligne du tableau $\mathrm{n}^{\circ} 1$ montre dans ce cas des coûts de fluctuation relativement faibles et comparables entre les systèmes d'assurance publique $(0,43 \%)$ et d'auto-assurance $(0,62 \%)$. Plus généralement, le coût du risque du chômage est considérablement réduit dans les deux cas par rapport à une situation dans laquelle les ménages ne disposeraient d'aucun mécanisme d'assurance, le coût des fluctuations s'élevant alors à 30,27\%. 


\section{Tableau 1}

\section{Coût des fluctuations de revenus}

\begin{tabular}{|lcc|}
\hline & $M C \rightarrow A C$ & $M C \rightarrow M F$ \\
\hline Etat stationnaire & 0,43 & 0,62 \\
Transition & 0,43 & 9,94 \\
Critère rawlsien & 18,02 & 83,60 \\
\hline
\end{tabular}

Coût en \% de consommation du passage d'une situation de marchés complets (MC) aux marchés incomplets (MF ou AC)

Ces premiers résultats pourraient laisser supposer que l'épargne de précaution est un moyen d'assurance alternatif aux allocations chômage. Cependant Algan et al. [2004] soulignent qu'une telle analyse masque la spécificité de chacun de ces instruments.

Tout d'abord, l'auto-assurance repose sur un processus d'accumulation préalable, c'est-à-dire sur une renonciation à la consommation pendant toute la phase de transition précédant l'arrivée au niveau de richesse d'état stationnaire. La deuxième ligne du tableau $\mathrm{n}^{\circ} 1$ montre que le coût des fluctuations dans une économie avec épargne de précaution augmente considérablement lorsqu'il est tenu compte de la transition, atteignant 9,6\% contre $0,73 \%$ dans les comparaisons d'état stationnaire. Le coût des fluctuations avec allocation chômage étant resté inchangé, la différence entre les deux mécanismes assurantiels est maintenant beaucoup plus importante. Une autre spécificité importante de ces mécanismes réside dans les inégalités entre individus qu'ils peuvent créer ou atténuer. Le niveau du stock d'épargne décroissant avec la durée du chômage, il peut exister de fortes inégalités assurantielles entre les individus. Les auteurs proposent de mesurer ces inégalités de position en adoptant un critère rawlsien privilégiant l'individu le plus démuni dans l'économie, c'està-dire ici une personne qui se retrouve à chaque période au chômage. La dernière ligne du tableau $n^{\circ} 1$ compare le coût des fluctuations pour cet individu sous les deux environnements. La différence devient alors plus importante puisque le coût des fluctuations pour le plus démuni passe de $18,02 \%$ dans l'envi- 
ronnement avec assurance chômage à 83,6 \% dans l'économie avec épargne de précaution. L'assurance chômage permet donc de limiter les inégalités de position et de pallier le rationnement du crédit pour les plus démunis.

Plus récemment Algan et Allais [2004] ont complété cette étude en prenant également en compte le cycle économique dans la comparaison du système d'épargne et du système d'assurance chômage. Deux facteurs supplémentaires peuvent alors jouer en défaveur du premier système. Le premier a trait aux fluctuations des prix et en particulier du taux d'intérêt. Il devient très coûteux d'épargner pendant les périodes où le rendement de l'épargne est faible. Le second facteur a trait à l'allongement de la durée moyenne du chômage pendant les périodes de récession. Si l'épargne de précaution peut être un bon substitut aux allocations pour financer de très courts épisodes de chômage, elle devient rapidement insuffisante lorsque la durée moyenne du chômage est de l'ordre de celle observée en France au cours des dernières décennies.

Cependant les analyses citées ci-dessus ne tiennent pas compte de l'impact des mécanismes assurantiels sur l'offre de travail. Cette question semble pourtant essentielle pour comparer les mérites respectifs de l'assurance chômage et de l'auto-assurance. La section suivante propose une synthèse des travaux relatifs à ce sujet.

\section{L'impact de l'épargne de précaution sur la recherche d'emploi}

Alors qu'il existe déjà une abondante littérature empirique consacrée à l'impact de l'assurance chômage sur l'offre de travail, ce n'est que très récemment que l'attention s'est portée également sur les effets de l'épargne de précaution. Pourtant ses effets théoriques ont été depuis longtemps établis par Danforth [1979]. Ce dernier montre que l'épargne partage les mêmes vertus assurantielles que les allocations en offrant la possibilité à un chômeur de rechercher plus longtemps la meilleure offre possible. Il en 
dégage trois propositions : les riches sont plus sélectifs dans leur recherche, la durée de leur épisode de chômage est en conséquence plus longue mais le rendement de leur recherche est aussi plus élevé.

Deux grands types de travaux ont proposé de mesurer cet effet théorique de l'épargne de précaution mis en évidence par Danforth [1979]. Le premier consiste à calibrer et simuler des modèles d'offre de travail (Low [1999]) ou de recherche d'emploi (Acemoglu et Shimer [2001] et Algan et al. [2003]) dans lesquels les ménages peuvent épargner mais font face à des contraintes d'endettement. Ces études montrent que l'épargne peut avoir un impact quantitatif significatif sur le comportement des travailleurs. Ainsi, Algan et al. [2003] montrent que l'épargne peut influencer la décomposition en bons et mauvais emplois dans l'économie française en affectant la durée de recherche d'emploi et donc la qualité des emplois trouvés. En particulier, les chômeurs possédant un stock d'épargne de précaution élevé peuvent rester sans emploi plus longtemps à la recherche du meilleur appariement. A l'inverse, les chômeurs pauvres et contraints sur le marché du crédit acceptent les premiers emplois venus et peuvent se trouver ainsi pris dans des trappes à pauvreté. La richesse initiale est donc susceptible de jouer un rôle fondamental d'hystérèse dans la mobilité professionnelle, ce qui n'est pas sans soulever de nouveaux débats sur la fiscalité optimale du patrimoine et de l'héritage.

Le second type de travaux consiste à donner des fondements empiriques aux modèles précédents, en estimant directement l'impact de l'épargne sur les stratégies de recherche d'emploi à partir de panels de données individuelles. Ces estimations ont été conduites sur les marchés du travail hollandais (Bloemen et Stancanelli [2001]) ou encore français (Algan et Terracol [2001]). Nous offrons dans cette section une synthèse des principaux résultats de ces derniers qui exploitent la base française du panel européen sur la période 1993-1996. Dans cette étude, l'auto-assurance des chômeurs est identifié à leurs livrets d'épargne qui sont étagés en trois niveaux : le niveau 1 (moins 
de 1524 euros), le niveau 2 (de 1524 à 4573 euros) et enfin le niveau 3 (plus de 4753 euros) ${ }^{2}$.

Les auteurs estiment tout d'abord l'impact de l'autoassurance sur le salaire de réserve horaire qui est déclaré par les chômeurs recensés dans le panel européen. Le tableau n ${ }^{\circ} 2$ présente les résultats de cette estimation (en log) en tenant également compte de toutes les caractéristiques traditionnelles telles que l'égibilité aux allocations chômage, le travail du conjoint, l'âge, le diplôme et le sexe du chômeur. Il apparaît dans ce cadre que l'épargne a toujours un impact positif sur le salaire de réserve. Cependant cette influence n'est statistiquement significative qu'à partir d'un livret niveau 3 (patrimoine supérieur à 4753 euros), ce qui ne concerne pas plus de $11 \%$ des chômeurs dans la base de données. Mais ce niveau est caractéristique puisqu'il correspond approximativement au niveau annuel moyen qu'un chômeur perçoit dans la base. L'épargne n'a donc d'impact sur la recherche d'emploi que pour des niveaux équivalents à ceux de l'assurance chômage.

Les auteurs procèdent également à l'estimation des effets de l'épargne de précaution sur la durée du chômage. Théoriquement, la hausse du salaire de réserve doit se traduire par une augmentation de la probabilité de refuser une offre et donc par un allongement de la durée du chômage. Pour évaluer la pertinence empirique de cette relation, les auteurs utilisent un modèle de durée dans lequel le taux mensuel de sortie du chômage est expliqué par le niveau d'épargne détenu au début de l'épisode, tout en tenant compte des autres variables traditionnelles et d'une hétérogénéité résiduelle inobservée entre les individus. Les estimations du modèle de durée, présentées dans le tableau n ${ }^{\circ} 3$, montrent que l'épargne diminue toujours le taux mensuel de sortie du chômage, à l'instar des allocations chômage. Cependant cet effet n'est statistiquement significatif qu'à partir d'un accroissement de richesse du niveau 1 vers le niveau 3 . La probabilité de sortir du chômage diminue alors de $23,1 \%$, ce qui est proche de la baisse de $26,9 \%$ occasionnée par la perception d'allocations chômage ${ }^{3}$. 
En conclusion, l'épargne de précaution a des effets de signe similaires à ceux de l'assurance chômage sur les comportements de recherche d'emploi. Mais cette influence n'est statistiquement significative qu'à partir de niveaux élevés de stocks d'épargne. Dans cette perspective, le dispositif d'assurance publique se révèle essentiel pour aider dans leur recherche d'emploi la grande majorité des chômeurs qui sont rationnés sur le marché du crédit.

\section{Epargne et niveau optimal des allocations chômage}

La prise en compte de l'épargne de précaution n'est naturellement pas neutre quant à l'évaluation traditionnelle du niveau optimal des allocations chômage. Les sections précédentes ont en effet souligné que l'épargne est susceptible d'offrir une assurance complémentaire aux allocations chômage et en partage les mêmes effets qualitatifs sur les stratégies de recherche d'emploi à partir d'un certain niveau de patrimoine. Il est donc essentiel de réévaluer le niveau optimal des allocations chômage à l'aune des comportements d'épargne des ménages. Le système d'assurance chômage optimal résulte toujours de l'arbitrage traditionnel entre le bénéfice assurantiel et le coût qu'elles génèrent en termes d'aléa moral sur l'effort de recherche des chômeurs. La littérature s'est alors proposé de réévaluer successivement le niveau optimal des allocations chômage puis leur profil optimal.

La première voie de recherche, inspirée par Hansen et Imrohoroglu [1992], se focalise sur le seul niveau optimal des allocations chômage. Elle s'inscrit dans le cadre de modèles d'équilibre général dans lesquels les ménages peuvent épargner. Le critère d'optimalité consiste alors uniquement à déterminer le niveau du taux de remplacement qui maximise le bien-être moyen à l'état stationnaire de l'économie. Cependant ces travaux se focalisent davantage sur le problème d'aléa moral que sur le rôle additionnel joué par l'épargne, cette dernière caractéristique étant introduite uniquement par souci de réalisme. Dans un 
modèle de recherche d'emploi partiel calibré sur les Etats-Unis, Hansen et Imrohoroglu [1992] montrent que le niveau optimal du taux de remplacement diminue radicalement de $65 \%$ à $15 \%$ en présence d'aléa moral.

La seconde voie de recherche reprend le cadre de contrat d'assurance, proposé par Shavell et Weiss [1979] $]^{4}$, dans lequel le gouvernement décide du niveau optimal des allocations chômage lorsqu'il n'observe qu'imparfaitement l'effort de recherche d'emploi du chômeur. Dans ce cadre canonique sans épargne, le gouvernement doit arbitrer entre des motifs d'assurance et d'efficacité. Le motif d'assurance incite le gouvernement à maintenir un niveau de consommation constant pour le chômeur et à proposer en conséquence un profil des allocations chômage qui soit constant. Cependant cette règle n'est pas nécessairement efficace dans la mesure où elle n'incite pas le chômeur à fournir un effort de recherche. Aussi le motif d'efficacité nécessite-t-il que le gouvernement propose un profil dégressif des allocations chômage, le chômeur étant incité à redoubler d'effort au fur et à mesure que son niveau de consommation diminue.

La prise en compte de l'épargne des travailleurs modifie-t-elle ce résultat classique de décroissance du profil optimal des allocations chômage avec la durée passée au chômage? Deux contributions se sont attaché à répondre à cette question dans le cadre de modèles à durée de vie infinie. La première réponse de Wang et Williamson [2002] s'inscrit dans le cadre où les autorités publiques peuvent observer le niveau d'épargne du travailleur. Dans ce cas, le résultat est assez trivial : les autorités publiques ont intérêt dans un premier temps à fixer un niveau de remplacement nul jusqu'à ce que le chômeur épuise son stock d'épargne puis à proposer, dans un second temps, le même type de taux de remplacement dégressif que Shavell et Weiss [1979]. La seconde réponse, apportée notamment par Werning [2002] et Kocherlakota [2004], est plus riche en ce qu'elle suppose que les autorités publiques ne peuvent observer le niveau d'épargne. Il existe donc un double aléa moral : sur l'effort de recherche et sur le niveau d'auto-assurance du chô- 


\section{Tableau 2}

\section{Estimation du salaire de réserve des chômeurs}

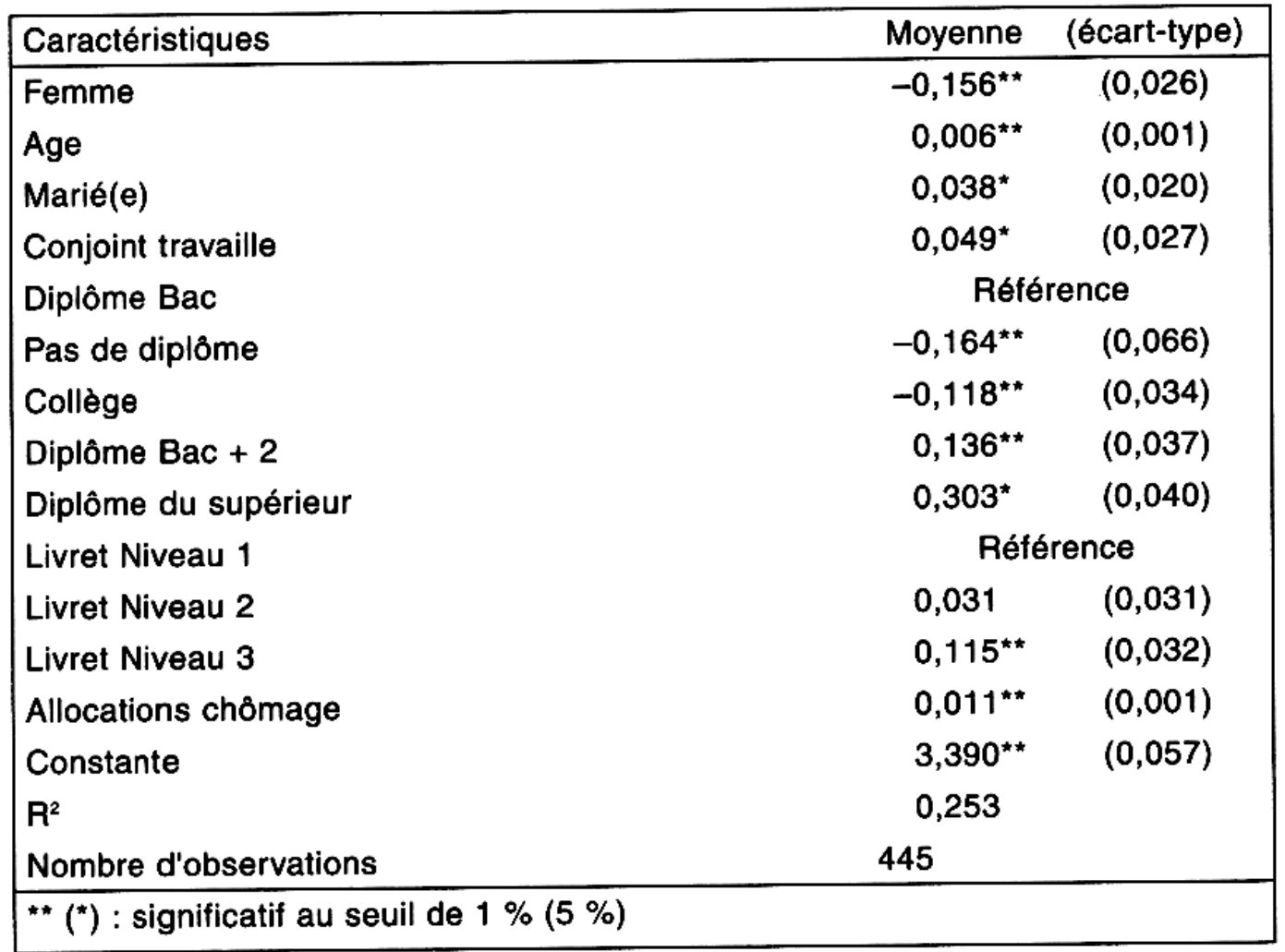

\section{Tableau 3}

\section{Estimation du taux de sortie du chômage}

\begin{tabular}{|c|c|c|}
\hline Variables & Coefficients & (écart-type) \\
\hline Femme & $-0,458^{\star \star}$ & $(0,096)$ \\
\hline Age & $-0,020^{* *}$ & $(0,005)$ \\
\hline Diplôme général & \multicolumn{2}{|c|}{ Référence } \\
\hline Aucun diplôme & $-0,571^{\star \star}$ & $(0,073)$ \\
\hline Diplôme technique & $0,014^{* *}$ & $(0,003)$ \\
\hline Diplôme supérieur & $0,011^{\star \star}$ & $(0,004)$ \\
\hline Allocations chômage & $-0,319^{\star \star}$ & $(0,078)$ \\
\hline Livret Niveau 1 & \multicolumn{2}{|c|}{ Référence } \\
\hline Livret Niveau 2 & $-0,163$ & $(0,117)$ \\
\hline Livret Niveau 3 & $-0,263^{\star \star}$ & $(0,082)$ \\
\hline Constante & $-0,867^{\star \star}$ & $(0,227)$ \\
\hline Log-Vraisemblance & $-1419,912$ & \\
\hline Nombre de durées mensuelles de chômage observées & 4717 & \\
\hline
\end{tabular}


meur. Dans ce cas, Werning [2002] montre que le profil optimal du taux de remplacement peut devenir croissant. Pour comprendre ce résultat, rappelons-nous que l'argument de Shavell et Weiss repose sur le fait qu'un système d'assurance dégressif induit un sentier de consommation décroissant. Mais dans un modèle avec épargne, l'allocation intertemporelle de la consommation dépend aussi bien des décisions du principal en termes de fixation du taux de remplacement que des décisions de l'agent en termes de choix d'épargne. Comme un agent désépargne au cours de l'épisode de chômage, il n'est pas nécessaire que le profil du taux de remplacement soit décroissant pour que la consommation de l'agent diminue. Le motif d'incitation lié à la décroissance du taux de remplacement peut donc être contrebalancé par le motif d'assurance.

En conclusion, la littérature relative aux comportements d'auto-assurance semble offrir deux pistes de réflexion principales sur la réforme de l'assurance publique. D'une part, les mécanismes d'auto-assurance accroîtraient fortement les inégalités en termes de couverture contre les risques de chômage par rapport à l'assurance publique. En effet, l'hétérogénéité dans les capacités d'auto-assurance est complètement conditionnée par l'hétérogénéité des trajectoires individuelles sur le marché du travail. Un mécanisme de redistribution publique semble donc indispensable pour ceux qui connaissent un parcours professionnel plus heurté. D'autre part, l'auto-assurance ne semble affecter que le comportement de recherche d'emploi des chômeurs les plus aisés. Les problèmes d'aléa moral ne se poseraient donc qu'aux chômeurs disposant d'un certain patrimoine et concernent peu les chômeurs contraints sur le marché du crédit. Ce résultat plaide pour un mécanisme de contrôle davantage ciblé sur certaines catégories de chômeurs plutôt qu'un système de contrôle indifférencié tel que celui proposé par le PARE. Cependant ces pistes sont susceptibles d'être enrichies par la prise en compte d'un autre type d'assurance complémentaire aux allocations chômage : l'assurance collective ou interpersonnelle. 


\section{Assurance chômage et assurance interpersonnelle}

\section{L'assurance interpersonnelle contre les risques de chômage}

L'importance empirique du phénomène d'assurance interpersonnelle

L'impact assurantiel des allocations chômage dépend, dans une large mesure, de l'intensité avec laquelle les agents se prémunissent individuellement contre les risques qu'ils encourent sur le marché du travail. Si dans ce cadre l'épargne individuelle joue un rôle primordial, la prise en compte de certains phénomènes sociaux demeure essentielle. La famille, les amis et le cadre élargi des relations constituent une source fondamentale d'assurance contre la baisse de consommation subie suite à la perte d'un emploi. De ce point de vue, il est important de noter que les agents peuvent se transférer à la fois des actifs traditionnels (aide alimentaire, financière etc.) mais aussi des actifs de type informationnels, par exemple des offres d'emploi. Ce dernier point, auquel nous consacrerons une section à part entière, a une importance empirique indéniable. Une série de travaux a montré qu'en moyenne $50 \%$ des individus obtenaient leur emploi grâce à leurs réseaux de relations (Granovetter [1995], Holzer [1988], Addison et Portugal [2001]).

Les agents, regroupés au sein de groupes sociaux (ce terme étant entendu dans un sens élargi), s'assurent donc mutuellement. Si l'un des membres du groupe vient à perdre son emploi, les autres membres opèrent des transferts limitant la perte initiale de bien-être. Cette assurance mutuelle est soutenable soit grâce à l'altruisme qui lierait les agents (Altonji et al. [1997]), soit parce que ne pas aider les individus subissant un choc négatif de revenu revient à s'exclure de ce mécanisme d'assurance (Attanasio, Ríos-Rull [2000]).

Bentolila et Ichino [2003] ont cherché à évaluer l'importance empirique de ce type d'assurance. Ils étudient dans un 
18 Yann Algan, Pierre Cahuc, Bruno Decreuse, François Fontaine, Solenne Tanguy

premier temps, sur un panel de cinq pays (Allemagne, Espagne, Etats-Unis, Grande-Bretagne et Italie), l'impact d'une augmentation de la durée du chômage sur la perte de consommation des ménages. Cette perte de consommation est ensuite rapportée à différents indicateurs. Certains représentent notamment l'intensité des liens interpersonnels entre les membres d'une famille (tableau $\mathrm{n}^{\circ} 4$ ).

Tableau 4

Indicateurs des liens familiaux

Notes : données pour l'année 1995 (1992 pour les Etats-Unis). N : taille de l'échantillon. Ecarts-types entre parenthèses.

Source : Bentolila et Ichino [2003].

Sur la base de ces indicateurs, les auteurs montrent que les liens familiaux sont plus forts au sud (Italie, Espagne) qu'au nord (Allemagne, Grande-Bretagne, Etats-Unis). En particulier, la fraction des ménages habitant avec un parent ou la part de ceux qui reçoivent une aide de leur famille donne une idée de l'importance des liens de réciprocité au sein de la famille. Ces statistiques sont significativement plus grandes au sud que dans les pays du nord. Parallèlement, leurs résultats empiriques indiquent qu'une augmentation de la durée du chômage conduit à des pertes en termes de consommation plus faibles dans les pays 
du sud qu'au nord. Ce dernier fait est difficilement explicable par les théories économiques traditionnelles. En particulier, une explication en termes de contrainte de liquidité ne serait être ici satisfaisante. En effet, les consommateurs étant plus largement contraints sur leurs liquidités dans des pays comme l'Espagne ou l'Italie que dans les pays du nord (Jappelli et Pagano [1989]), le niveau de leur consommation devrait répondre beaucoup plus fortement à un allongement de la durée du chômage. On pourrait aussi penser que l'existence de systèmes d'assurance chômage plus généreux au sud constitue une explication valable du phénomène observé. Néanmoins, et malgré l'exercice difficile que constitue ce genre de comparaisons, un examen détaillé des systèmes d'assurance chômage montre qu'ils n'y sont nullement plus avantageux (tableau $\mathrm{n}^{\circ} 5$ ).

Si les ratios de remplacement paraissent plus élevés pour l'Italie et l'Espagne les six premiers mois, il en va tout autrement pour des durées plus importantes de chômage, cette distinction étant d'autant plus nécessaire que la part des chômeurs de long terme est plus importante dans ces pays. Globalement, le taux de couverture de l'assurance chômage y est plus faible.

\section{Tableau 5}

Systèmes d'assurance chômage (1990-1995) 
Bentolila et Ichino passent ainsi en revue les théories utilisées généralement dans l'étude du marché du travail. Devant leur incapacité à expliquer le phénomène observé et du fait de l'importance empirique constatée des réseaux de relations (ici essentiellement familiaux), ils concluent que si la perte de consommation des chômeurs, suite à un allongement de la durée du chômage, est moins importante au sud qu'au nord, c'est avant tout parce que le mécanisme d'assurance interpersonnelle y est plus présent.

Le phénomène d'assurance interpersonnelle est donc empiriquement significatif. Même dans les pays du nord, il est susceptible d'interagir avec le système d'assurance publique (voir tableau $\mathrm{n}^{\circ} 4$ ) : on remarquera que dans un pays comme les EtatsUnis, la probabilité de recevoir une aide de la famille suite à la perte de son emploi est importante. Un système public aurait donc d'autant moins d'impact sur le bien-être que les liens sociaux entre les individus sont forts.

\section{Quelles interactions entre les systèmes publics et privés d'assurance?}

Cependant, il est probable que l'existence et le développement de l'assurance chômage publique interagissent avec ce mécanisme d'assurance interpersonnelle. Attanasio et Ríos-Rull [2000] ont notamment montré que le renforcement ou la mise en place d'un système d'allocation pouvait mettre à mal les liens sociaux existants. Etudiant l'impact d'un vaste système d'aides sociales (santé, nourriture et éducation) pour des populations rurales au Mexique en 1997 (programme PROGRESSA), ils montrent que ce programme a entraîné une diminution significative des transferts monétaires entre agents. Au final, il se serait révélé incapable d'élever le niveau de vie des agents du fait de l'existence d'un effet d'éviction entre les transferts publics et les transferts interpersonnels.

Les transferts privés entre les individus ne sont soutenables que parce que les agents ont intérêt à se prémunir contre les risques futurs qu'ils encourent s'ils perdent eux aussi leur emploi. En quelque sorte, pour être aidé, il faut d'abord avoir aidé, avoir 
établi un lien de réciprocité soutenable, d'obligations mutuelles dont la transgression conduit à des sanctions. Plus ces risques sont forts, ou plus la perte de revenu en cas de licenciement est importante, et plus l'engagement qui lie les agents les uns aux autres est fort. Lorsque le niveau des allocations chômage augmente, la situation des chômeurs s'améliore, indépendamment du fait qu'ils participent ou non au système d'assurance interpersonnelle. Par conséquent, plus un système d'assurance publique est généreux et plus les individus ont un intérêt réduit à aider ceux qui subissent une dégradation de leur situation sur le marché du travail. D'un côté les allocations chômage améliorent, toutes choses égales par ailleurs, la situation des chômeurs, mais d'un autre côté l'effet d'éviction sur les transferts privés rend l'impact final en termes de bien-être ambigu. Ce mécanisme pourrait expliquer également pourquoi les pays du nord, où les systèmes d'assurance chômage sont sous certains aspects plus généreux, sont caractérisés par des systèmes sociaux d'assurance interpersonnels moins importants (voir tableaux $\mathrm{n}^{\text {os }} 4$ et 5 ).

Quelles sont les conséquences de cet effet d'éviction de l'assurance publique sur l'assurance interpersonnelle ? Comme le soulignent Bentolila et Ichino, les réseaux familiaux ou relationnels sont généralement d'une taille limitée et concentrés géographiquement et sectoriellement. Les travaux économétriques de Topa [2000] ont ainsi montré que l'existence de réseaux de relations conduit à une corrélation spatiale positive des taux de chômage, cette corrélation étant d'autant plus forte que les zones étudiées sont peu éduquées et comportent une fraction importante de minorités. Dans le cas d'un choc négatif affectant spécifiquement un type d'industrie, une région ou un type de qualification, ils ne constituent pas une source satisfaisante d'assurance du fait d'une insuffisante diversification des risques, tous les agents du groupe social ou familial étant affectés en même temps. Leur efficacité se limite donc aux chocs idiosyncratiques et ils ne sauraient se substituer à l'assurance publique lors de chocs agrégés.

La prise en compte des transferts privés semblerait par conséquent limiter la nécessité d'un système d'allocations chô- 
mage. Cependant, les agents tendent à se lier avec des individus proches en termes de caractéristiques sociales (Granovetter [1995]) et ce biais constitue une justification supplémentaire des systèmes publics. L'effet d'éviction présenté doit néanmoins être gardé à l'esprit lorsque l'on considère une augmentation des allocations et plus largement de toute aide sociale. En outre, tout porte à croire que cet effet d'éviction est d'autant plus fort que les individus sont initialement peu protégés. Dans ce cas, un renforcement de l'assurance chômage doit être accompagné d'une évaluation empirique précise de l'évolution des consommations des agents concernés.

\section{Interactions sociales et niveau optimal des allocations chômage}

\section{Le rôle des normes sociales}

Tout changement de statut sur le marché du travail implique un changement de statut social. Celui-ci peut être plus ou moins bien perçu en fonction des a priori du groupe social de référence. De même, le recours à l'assurance chômage et aux systèmes d'assistance est conditionné à la fois par la connaissance qu'ont les agents de leur existence et fonctionnement, et par la perception qu'a l'entourage de ces systèmes (Bertrand et al. [2000]). En particulier, le fait de recevoir des allocations et d'avoir recours aux agences publiques pour l'emploi peut être mal perçu et socialement stigmatisant. L'importante valorisation sociale faite par certains groupes de l'activité laborieuse rend les périodes de chômage particulièrement pénibles. Ceux qui dévient de cette norme que constitue le fait de travailler subissent alors des sanctions par l'intermédiaire d'une pression sociale plus ou moins forte et de l'exclusion à certaines activités du groupe (Boudon et Bourricaud [1994]). En termes économiques, cette exclusion fait que le chômeur ne peut valoriser de manière satisfaisante le loisir dont il dispose, ce qui affecte son bien-être.

Dans cette perspective, les problèmes traditionnels de risque moral doivent être relativisés et il est probable que l'am- 
pleur des allocations ne soit pas le seul déterminant de l'intensité de recherche des chômeurs. Un individu au chômage aura d'autant plus intérêt à faire un effort de recherche que les normes de son groupe social de référence valorisent l'activité productive (pénalisent socialement les situations de chômage). Stutzer et Lalive [2002] ont cherché à évaluer l'impact des normes sociales sur les taux de sortie du chômage. L'une de leurs contributions est d'avoir offert une nouvelle mesure des normes sociales en liant les choix électoraux des individus à leurs croyances. En 1997, un référendum fut initié en Suisse pour savoir si les allocations chômage devaient être diminuées. Rapidement, le débat public porta sur une évaluation empirique du chômage volontaire, mais il fut aussi la scène d'une série de jugements moraux sur les chômeurs. Le non l'emporta finalement d'une courte majorité et le niveau des allocations resta inchangé. Une série d'enquêtes (réalisées auparavant ou directement à la sortie des urnes) a montré que le vote des électeurs avait été fortement lié aux normes sociales intériorisées. Stutzer et Lalive montrent notamment la corrélation importante pour chaque canton entre le pourcentage d'individus ayant voté pour la baisse des allocations et le pourcentage d'individus affirmant être d'accord avec l'affirmation "les individus qui ne vivent pas de leurs propres revenus sont inutiles. » Cette corrélation permet de justifier l'idée selon laquelle les votes exprimés reflètent les normes sociales : plus un district aura été favorable à la baisse des allocations et plus les normes sociales doivent y être considérées comme défavorables vis-à-vis des chômeurs.

Dans un second temps, les auteurs testent la relation entre le vote exprimé dans chaque district et la durée du chômage, i.e. entre les normes sociales et les taux de retour à l'emploi. Afin de saisir de manière adéquate le sens de la causalité, la durée moyenne du chômage est évaluée sur un très large échantillon d'individus devenus chômeurs six mois après le vote. Cet échantillon, qui représente $85 \%$ de l'échantillon exhaustif, est ensuite suivi pendant un an et demi. L'étude économétrique, assez complète et prenant en compte les problèmes d'estimation propres aux phénomènes d'interaction sociale ${ }^{5}$, permet à Stutzer et Lalive 
24 Yann Algan, Pierre Cahuc, Bruno Decreuse, François Fontaine, Solenne Tanguy

d'effectuer des simulations numériques. Celles-ci permettent à la fois d'évaluer l'importance quantitative de la relation entre les normes sociales et la durée du chômage, et de comparer l'effet d'un renforcement des normes (i.e. d'une plus forte stigmatisation des chômeurs) à une variation du taux de remplacement (tableau $\mathrm{n}^{\circ}$ 6).

On remarquera tout d'abord que la médiane de la durée du chômage se situe à 140 jours. Une hausse du taux de remplacement de $10 \%$ (de $70 \%$ à $80 \%$ ) augmente la durée du chômage de plus de 12 jours pour un individu ayant des caractéristiques moyennes par rapport à l'échantillon utilisé. Ce résultat est conforme à celui de diverses études économétriques. Une variation positive de l'intensité de la norme sociale correspondant à la valeur de l'écart-type réduit quant à elle cette durée de plus de 11 jours ${ }^{6}$. Les normes sociales semblent donc avoir potentiellement autant d'impact que le niveau des allocations chômage. Le rôle qu'elles peuvent jouer dans les problèmes de risque moral est donc tout à fait fondamental. Une communauté sanctionnant socialement les périodes longues de chômage constitue une limite aux stratégies de recherche d'emploi inefficaces ou insuffisamment intensives. Pour de tels groupes sociaux, l'effet désincitatif sur la recherche d'emploi des allocations chômage est quantitativement de faible importance et le contrat à leur proTableau 6

Simulations de l'importance quantitative des normes sociales sur la durée du chômage

Notes : les simulations se réfèrent à un individu qui aurait, par rapport à l'échantillon de référence, des caractéristiques moyennes. L'intensité de la norme sociale correspond au pourcentage de personnes en faveur de la réduction des allocations chômage.

Source : Stutzer et Lalive [2002]. 
poser ne doit plus tant insister sur l'aspect incitatif que sur l'aspect assurantiel.

L'analyse des systèmes assurantiels et incitatifs nécessite donc une compréhension profonde du milieu social sur lequel ils agissent. De nombreux sociologues ont montré que l'existence de réseaux sociaux permet le développement et la reproduction d'une " culture de la pauvreté " (Wilson [1987]). En son sein, les réseaux servent de relais à des informations précises sur l'utilisation des systèmes assurantiels et d'assistance, au lieu de jouer leur rôle traditionnel de transmetteurs d'offres d'emploi. Cette " culture de la pauvreté ", qui concerne des groupes sociaux bien délimités, joue un effet désincitatif sur la recherche d'emploi (Bertrand et al. [1998]). Tout d'abord elle correspond à des normes sociales valorisant peu l'activité productive. Ensuite, la connaissance des aides sociales disponibles ou de l'assurance chômage affaiblit les incitations au retour à l'emploi. Ce constat invite donc à concentrer les systèmes de conseils et de contrôle sur la partie de la population la plus défavorisée, donc la plus susceptible d'adopter cette culture de la pauvreté. Ceci contredit l'idée évoquée plus haut selon laquelle le contrôle devrait être ciblé sur les catégories de chômeurs les plus aisés c'est-à-dire les moins contraints sur le marché financier.

Par ailleurs, de nombreux travaux sur les allocations chômage naissent d'une comparaison empirique Europe/Etats-Unis qui n'est pas toujours pertinente. En effet, l'impact d'un système assurantiel sur le bien-être des individus est fortement corrélé avec la perception qu'ont les individus des inégalités qui les entourent. Un système d'assurance chômage affaibli conduit a priori à un niveau d'inégalités plus important puisque les agents se voient obligés de passer par des systèmes moins efficaces (assurance interpersonnelle), plus coûteux et supposant un niveau de revenu suffisant (épargne privée). Or, Alesina et al. [2001] ont montré qu'il existe un lien empiriquement significatif entre le sentiment de bien-être des individus et le niveau des inégalités. Les individus ont, toutes choses égales par ailleurs, un bien-être d'autant plus faible qu'ils vivent dans une société inégalitaire. En effet, l'existence d'inégalités peut, par exemple, aller à l'encontre de cer- 
taines normes sociales intériorisées. Les auteurs montrent, en outre, que ce lien entre niveau des inégalités et bien-être est plus fort en Europe qu'aux Etats-Unis. L'évaluation du système d'allocations en termes de bien-être social doit prendre en compte ces différences. A la lumière de ces résultats, on peut supposer qu'il devra être plus égalitaire en Europe et que son affaiblissement, par exemple en vue de favoriser l'auto-assurance par l'épargne, aurait des conséquences fortement négatives sur le bien-être.

\section{Méthodes de recherche et interactions sociales}

La prise en compte de l'existence d'un système d'assurance interpersonnelle est susceptible d'enrichir l'étude du contrat d'assurance optimal. En effet, ce nouvel élément vient modifier assez profondément l'arbitrage traditionnel entre assurance et incitation. De ce point de vue, ce n'est pas seulement d'un problème d'incitation à l'effort qu'il s'agit mais aussi d'incitation à l'utilisation de méthodes de recherche efficaces. Agences publiques ou privées pour l'emploi, candidatures spontanées ou annonces dans la presse spécialisée sont généralement qualifiées de méthodes formelles. L'analyse économique n'a longtemps considéré que ces seules méthodes, omettant celle qui empiriquement est la plus utilisée : les réseaux de relations (famille, amis, relations profes-

Tableau 7

Méthodes de recherches utilisées par les individus déclarant chercher un emploi et ayant fait des démarches dans ce sens

Notes : plusieurs méthodes peuvent être utilisées simultanément. Echantillon : 10976 individus.

Source : enquête emploi INSEE 1998. 
sionnelles...) ou " méthode informelle ». De nombreux travaux ont montré qu'en moyenne $50 \%$ des emplois étaient trouvés grâce aux réseaux (Granovetter [1995], Montgommery [1991], Holzer [1988]). De même $40 \%$ des entreprises utiliseraient consciemment les réseaux de relations de leurs employés (Holzer [1987], Campbell et Mardsen [1990]). Les données fournies pour l'enquête sur l'emploi de l'Insee [1998] permettent, dans une certaine mesure, de donner une image statistique de ce phénomène pour le cas français (tableaux $\mathrm{n}^{\text {s }} 7$ et 8 ). En outre, on remarquera que les résultats ici présentés sont conformes à ce qui a pu être trouvé pour d'autres pays (Addison et Portugal [2001]).

Si les individus utilisent simultanément plusieurs méthodes durant leur recherche d'emploi, l'utilisation des réseaux de relations semblent très largement répandue (tableau $\mathrm{n}^{\circ} 7$ ), juste derrière l'agence publique pour l'emploi. Cette dernière bénéficie de l'obligation de s'y inscrire pour toucher les allocations chômage.

En ce qui concerne l'efficacité des différentes méthodes de recherche, ce sont les démarches personnelles auprès de l'entreprise et les réseaux qui obtiennent le plus grand succès. On remarquera que sur ce point l'enquête est fort imprécise et que certains enquêtés ont pu considérer avoir opéré une " démarche personnelle auprès de l'entreprise " tandis que leur offre avait été transmise grâce à un contact. En effet, l'utilisation de réseaux ne correspond pas nécessairement à l'intervention d'une relation auprès de l'entreprise. Il suffit que l'information sur le poste vacant ait été communiquée par une relation, ce qui est rarement pris en compte par les personnes interrogées. En outre, on remarque que l'efficacité des réseaux ne dépend pas significativement du type de poste à pourvoir, contrairement aux agences pour l'emploi qui véhiculent avec succès essentiellement les offres concernant les postes les moins qualifiés.

Si les réseaux sociaux assurent les individus par l'intermédiaire de transferts financiers ou matériels, ils le font aussi en accélérant, à moindre coût, la recherche d'emploi des personnes au chômage. Cependant, rien n'assure que de telles méthodes de recherche peu onéreuses soient efficaces pour l'ensemble de la 
28 Yann Algan, Pierre Cahuc, Bruno Decreuse, François Fontaine, Solenne Tanguy

Tableau 8

Méthode de recherche ayant été utilisée pour trouver le poste actuellement occupé chez les individus travaillant depuis moins d'un an dans leur entreprise

Notes : les types de poste correspondent à la position professionnelle de l'emploi actuel. Type 1: manœuvre, ouvrier spécialisé, employé de catégorie C ou D de la fonction publique. Type 2 : ouvrier qualifié ou hautement qualifié, agent de maîtrise, technicien, VRP (non cadre). Type 3 : ingénieur, cadre (hors fonction publique). Source : enquête emploi INSEE 1998.

société. Dans cette perspective, la présence d'une assurance publique doublée d'instruments de contrôle peut avoir des effets bénéfiques. L'existence d'allocations chômage et de systèmes de sanctions modifie la stratégie des agents, en ce qui concerne à la fois l'intensité de leur recherche mais aussi la répartition de cet effort sur les différentes méthodes de recherche. Van den Berg et van der Klaauw [2001] et Cahuc et Fontaine [2002] ont cherché à comprendre les interactions entre les différents systèmes d'assurance (assurance publique et réseaux sociaux) et les stratégies de recherche d'emploi des individus.

Van den Berg et van der Klaauw [2001] ont montré, sur données néerlandaises, qu'il existe une substitution entre les méthodes de recherche d'emploi. En particulier, il apparaît qu'un système d'allocations chômage avec contrôle de la recherche incite bien les agents à substituer à l'utilisation des méthodes informelles des méthodes formelles observables par l'agence pour l'emploi et leur assurant un paiement de leurs allocations.

Cahuc et Fontaine [2002] étudient les conséquences de ce phénomène de substitution dans une économie où les agents peuvent utiliser deux méthodes : une première, efficace mais 
coûteuse, est qualifiée de formelle, une autre, peu coûteuse mais moins efficace, repose sur les réseaux de relations. Sur ce point, il convient de distinguer l'efficacité ex ante et l'efficacité ex post d'une méthode de recherche. Ex ante, la méthode de recherche la plus efficace est celle qui génère, toutes choses égales par ailleurs, le taux de rencontres travailleur-employeur le plus important. Malgré son efficacité ex ante, une méthode peut se révéler ex post inefficace si peu d'agents l'utilisent à l'équilibre. Ainsi, les demandeurs d'emploi utiliseront-ils les méthodes formelles sans succès si peu d'entreprises prennent en considération les candidatures spontanées ou utilisent les agences pour l'emploi. Dans cette optique, le succès apparent des réseaux de relations dans la recherche d'emploi ( $c f$. tableau $n^{\circ} 8$ ) témoigne avant tout de son utilisation systématique des deux côtés du marché et laisse ouverte la question de l'optimalité des choix de méthode de prospection.

Les agents choisissent l'une ou l'autre des méthodes en évaluant les gains et les coûts associés à chacune. A l'équilibre, tous les agents utilisent, dans ce cadre simplifié, la même méthode. Si un individu peut évaluer les gains individuels de ses actions, celles-ci sont généralement entreprises sans prendre en compte leur impact sur les autres agents (leurs stratégies de recherche sont considérées comme une donnée) et plus largement sur le bienêtre social. Dans ce domaine comme dans d'autres, l'équilibre décentralisé peut être socialement inefficace : les agents adoptent une méthode inefficace étant données les caractéristiques de l'économie.

Notamment, les agents peuvent choisir d'utiliser leurs réseaux malgré leur inefficacité sociale. La raison principale est leur faible coût. Dans ce cadre la mise en place d'un système d'allocations conditionnelles à la méthode utilisée est possible. On remarquera que les méthodes formelles donnent généralement lieu à des actions observables par l'agence. Celle-ci peut donc conditionner l'obtention des allocations à l'acceptation par les chômeurs de rendez-vous d'embauche, ou encore à l'envoi régulier de candidature sur des offres proposées par l'agence. En outre, elle devra proposer des allocations suffisamment importantes pour 
couvrir le surcoût engendré par l'utilisation des méthodes formelles. Il semble également nécessaire de subventionner la recherche des deux côtés du marché du travail. Pour sortir d'un équilibre inefficace, il faut inciter les firmes à envoyer leurs offres aux agences publiques pour l'emploi. Des subventions, couvrant les coûts supplémentaires engendrés par l'utilisation de ce canal, permettent d'encourager les firmes à chercher de nouveaux employés grâce aux méthodes formelles. L'agence pour l'emploi ${ }^{7}$ devient alors un organe de coordination des demandeurs et des offreurs de travail sur le marché. Elle ne se bornerait pas à jouer un rôle d'assurance mais permettrait de coordonner les agents sur les méthodes de recherche les plus efficaces.

On remarquera que Cahuc et Fontaine supposent dans leur analyse que les agents ne peuvent utiliser qu'une méthode à la fois. Cette hypothèse constitue une limite évidente du modèle. Néanmoins, les mécanismes décrits ici pourraient s'étendre, au prix d'une plus grande complexité analytique, au cas où les agents ont à répartir leur effort de recherche entre différentes méthodes. Sans exclure totalement les méthodes formelles, ils pourraient, dans certains cas, ne pas suffisamment les utiliser. Comme précédemment, il reviendrait à l'agence pour l'emploi d'inciter à une répartition efficace des méthodes ${ }^{8}$.

La prise en compte de l'existence de l'assurance interpersonnelle vient, comme l'auto-assurance, enrichir notre perception des conséquences de l'assurance publique. Assurance interpersonnelle et assurance chômage, qui apparaissent de prime abord partiellement substituables, ont en réalité des vertus assurantielles extrêmement différentes. Les réseaux sociaux, fortement concentrés géographiquement et sectoriellement, ne constituent pas une source satisfaisante d'assurance du fait d'une diversification des risques insuffisante. En outre, la part de chaque système d'assurance affecte les stratégies de recherche des agents. Les réseaux sociaux, en tant que source d'information peu coûteuse, peuvent inciter les agents à utiliser des méthodes de recherche d'emploi informelles inefficaces. Dans cette perspective, les allocations chômage permettent de subventionner une recherche basée sur des méthodes d'emploi formelles, a priori socia- 
lement plus efficaces, mais plus coûteuses. En outre, les normes sociales véhiculées par ces réseaux peuvent venir amoindrir l'effet désincitatif des allocations chômage. Notre vision du contrat d'assurance optimal s'en trouve largement modifiée.

Les travaux récents sur l'assurance chômage montrent que son étude ne peut faire l'économie de la prise en compte des systèmes d'auto-assurance et d'assurance interpersonnelle. Un résultat général est que ces systèmes ne peuvent se substituer à l'assurance publique puisque leurs propriétés assurantielles different largement. En outre, ces nouvelles réflexions, qui demandent à mûrir pour être pleinement opérationnelles, permettent de distinguer trois points d'ancrage importants pour améliorer l'efficacité de l'assurance chômage publique. Premièrement, il apparaît que la dégressivité du niveau des allocations chômage avec la durée du chômage n'est vraisemblablement pas nécessaire lorsque les individus peuvent épargner. Les périodes de chômage, où les individus désépargnent, induisent à elles seules un profil de consommation décroissant qui fournit les incitations suffisantes à la recherche d'un emploi. Deuxièmement, les travaux empiriques indiquent que les normes sociales, qui forgent les attitudes face au travail, peuvent influencer significativement les comportements de recherche d'emploi. Une meilleure connaissance de ce phénomène permettrait sans doute d'améliorer les mesures d'accompagnement et d'aide à la recherche d'emploi des chômeurs, notamment en ciblant plus particulièrement cer- 
32 Yann Algan, Pierre Cahuc, Bruno Decreuse, François Fontaine, Solenne Tanguy

taines populations où la pression sociale du groupe ne joue plus son rôle incitatif. Enfin, les réflexions sur le rôle de coordination du système d'allocation chômage indiquent qu'il est peut être souhaitable de subventionner des méthodes de recherche d'emploi formelles non seulement du côté des travailleurs, mais aussi du côté des employeurs.

Cet article présente et met en perspective des résultats obtenus par les chercheurs d'EUREQua dans le cadre de la convention "Déterminants et conséquences macro-économiques de la protection sociale "financée par la mission recherche du ministère de l'Emploi et de la solidarite (MIRE). Les auteurs remercient la MIRE pour son soutien financier, Pierre Morin ainsi que les deux rapporteurs anonymes.

Yann Algan est professeur d'économie, EUREQua, IZA.

Adresse : EUREQua-CNRS-université Paris 1, IZA, Email : yanalgan@univ-paris1.fr

Pierre Cabuc est professeur d'économie, CREST, IZA, CEPR.

Adresse : EUREQua-CNRS-université Paris 1, CREST, IZA, CEPR et Institut Universitaire de France,Email : cabuc@univ-paris1.fr.

Bruno Decreuse est professeur d'économie, EUREQua.

Adresse : EUREQua-CNRS-université Paris 1, Email : bruno.decreuse@univ-parisI.fr.

François Fontaine est moniteur, université Paris 1 Panthéon-Sorbonne.

Adresse : EUREQua-CNRS-université Paris 1, Email : francois.fontaine@univ-paris1.fr.

Solenne Tanguy est moniteur, université Paris 1 Panthéon-Sorbonne.

Adresse : EUREQua-CNRS-université Paris 1,Email : solenne.tanguy@univ-paris1.fr. 


\section{Notes}

1. L'épargne de précaution se définit formellement comme le supplément d'épargne généré par une situation d'incertitude par rapport à une situation sans aléa de revenus (Leland [1968]). En ce sens elle se distingue des simples motifs d'épargne de cycle de vie.

2. La base initiale comprend quatre types d'actifs : livret d'épargne, épargne logement, valeurs mobilières et produits d'assurance vie. Cependant seul le premier type d'actif, de par sa plus grande liquidité, s'est avéré être utilisé comme un instrument de financement des périodes de chômage par les ménages recensés dans la base. Cet actif, étagé initialement en huit niveaux, a été reconstruit en trois niveaux faute de données pour les patrimoines plus élevés chez les chômeurs (Algan et Terracol [2001]).

3. Dans le cadre des modèles à hasard proportionnel, les coefficients estimés mesurent l'impact multiplicatif de chaque variable sur le ratio du hasard de base. La forme exponentielle nécessite une transformation afin de pouvoir quantifier l'impact des caractéristiques individuelles. Ainsi le chiffre de 23,1\% a été calculé de la façon suivante. L'ac- croissement du niveau 1 au niveau 3 multiplie le hasard de base par exp $\{-0,263\}=0,769$. Il diminue alors de $23,1 \%(=1-0,769)$ le taux de hasard de base par rapport à une personne dont la caractéristique financière est ignorée.

4. Hopenhayn et Nicolini [1997] enrichissent ce résultat en tenant compte de la possibilité de taxer le travailleur quand ce dernier a retrouvé un emploi en fonction de la durée de son épisode de chômage précédent.

5. Sur ce point, le lecteur est invité à se référer à Manski [1993].

6. On notera que les auteurs offrent des estimations complémentaires venant confirmer que ce lien entre normes et durée du chômage passe bien par des phénomènes de sanctions sociales.

7. Le terme « agence pour l'emploi » doit être ici entendu au sens large et recouvre des réalités diverses selon les pays. Dans le cas français, nous ne faisons pas de distinctions explicites entre ANPE, Unedic et Assedic.

8. On remarquera, qu'il n'y a pas alors de raison a priori d'exclure totalement l'une des méthodes à l'optimum. 
34 Yann Algan, Pierre Cahuc, Bruno Decreuse, François Fontaine, Solenne Tanguy

\section{Références}

D. Acemoglu et R. Shimer [1999] : Efficient Unemployment Insurance, Journal of Political Economy, vol. 107, pp. 893923.

D. Acemoglu et R. Shimer [2000] : Productivity Gains from Unemployment Insurance, European Economic Review, vol. 44, pp. 1195-1224.

J. Addison et P. Portugal [2002] : Job Search Methods and Outcomes, Oxford Economic Papers, vol. 54(3), pp. 505533.

S. Aiyagari, R. [1994] : Uninsured Idiosyncratic Risk and Aggregate Saving, Quarterly Journal of Economics, vol. 109, pp. 659-684.

A. Alesina, R. Di Tella et R. MacCulloch [2003] : Inequality and Happiness : Are Europeans and Americans Different, à paraître in Journal of Public Economics.

Y. Algan et A. Terracol [2001]: L'influence de l'épargne de précaution sur la recherche d'emploi, Economie et statistique, vol. 349, pp. 63-77.

Y. Algan, A. Chéron, J.O. Hairault et F. Langot [2003] : Wealth Effect on Labor Market Transitions, Review of Economic Dynamics, vol. 6, pp. 156-178.

Y. Algan, A. Chéron, J.O. Hairault et F. Langot [2004] : Epargne de précaution et chômage : une évaluation quantitative de l'auto-assurance, à paraître in Annales d'économie et de statistiques.

Y. Algan et O. Allais [2004]: Incomplete Unemployment Insurance under Aggregate Fluctuations, à paraître dans Economics Bulletin.
J. Altonji, F. Hayashi et L. Kotlikoff [1997] : Altruism and Inter Vivos Transfers : Theory and Evidence, Journal of Political Economy, vol. 105, pp. 11211166.

J. Attanasio et J.-V. Ríos-Rull [2000] : Consumption Smoothing in Island Economies : Can Public Insurance Reduce Welfare?, European Economic Review, vol. 44, pp. 1225-1258.

S. Bentolila et A. Ichino [2003] : Unemployment and Consumption : Are Job Losses Less Painful Near the Mediteranean? CEPR discussion paper $n^{\circ} 2539$. www.cepr.org.

M. Bertrand, S. Mullainathan et E. Luttmer [2000]: Network Effects and Welfare Cultures, Quarterly Journal of Economics, vol. 115(3) : pp. 1019-1055.

O. Blanchard et J. Tirole [2003] : Protection de l'emploi et procédures de licenciement, rapport du Conseil d'analyse économique, La Documentation française.

H.G. Bloemen et E.G. Tancanelli [2001] : Individual Wealth, Reservation Wages, and Transitions into Employment, Journal of Labor Economics, vol. 19, pp. 401-439.

R. Boudon et F. Bourricaud [1994] : Conformité et déviance, in Dictionnaire critique de la sociologie ( $4^{e}$ éd.), Presses universitaires de France.

M. Browning et T.F. Crossley [2001] : Unemployment Insurance Benefits Levels and Consumption Changes, Journal of Public Economics, vol. 80, pp. 1-23.

P. Cahuc et F. Fontaine [2002]: On the efficiency of Job Search with Social Net- 
works, CEPR discussion paper $\mathrm{n}^{\circ} 3511$. www.cepr.org.

K. Campbell et P. Mardsen [1990] : Recruitment and Selection Processes: The Organizational Side of Job Searches, in Breiger, R. (ed.), Social Mobility and Social Structure, New York : Cambridge University Press, pp. 59-79.

H. Chiu et E. Karni [1998] : Endogenous Adverse Selection and Unemployment Insurance, Journal of Political Economy, vol. 106, pp. 806-827.

J.P. Danforth [1979] : On the Role of Consumption and Decreasing Absolute Risk Aversion in the Theory of Job Search, in S.A Lippman et J. McCall, eds, Studies in the Economics of Search, NorthHolland, New York, pp. 109-131.

A. Deaton [1991] : Saving and Liquidity Constraints, Econometrica, vol. 59, pp. 1221-1248.

E.M. Engen et J. Gruber [2001] : Unemployment Insurance and Precautionary Saving, Journal of Monetary Economics, vol. 47, pp. 545-579.

M. Granovetter [1995] : Getting a Job : A Study of Contacts and Careers ( $2^{\mathrm{e}}$ éd.), Harvard University Press, Cambridge.

G. Hansen et A. Imrohoroglu [1992] : The role of Unemployment Insurance in an Economy with Liquidity Constraints and Moral Hazard, Journal of Political Economy, vol. 100, pp. 118-142.

H.J. Holzer [1987] : Hiring Procedures in the Firm: Their Economics Determinants and Outcomes, Human Ressources and Firm Performance, ed. by Richard Block et al., Industrial Relations Research Association.

H. Holzer [1988] : Search Methods Use by Unemployed Youth, Journal of Labor Economics, vol. 6, pp. 1-21.

H.A. Hopenhayn et J.P. Nicolini [1997] : Optimal Unemployment Insurance, Jour- nal of Political Economy, vol. 105, pp. 412-438.

R.G. Hubbard, J. Skinner et S.P. Zeldes [1995] : Precautionary Saving and Social Insurance, Journal of Political Economy, vol. 103, pp. 360-399.

T. Jappelli et M. Pagano [1989] : Comsumption and Capital Market Imperfections : an International Comparison, American Economic Review, vol. 79, pp. 1088-1105.

J. Kiander [1993] : Endogeneous Unemployment Insurance in a Monopoly Union Model when Job Search Matters, Journal of Public Economics, vol. 52, pp. 105115.

N. Kocherlakota [2004] : Figuring out the Impact of Hidden Savings on Optimal Unemployment Insurance, à paraître dans Review of Economic Dynamics.

E. Leland [1968] : Saving and Uncertainty : the Precautionary Demand for Saving, Quarterly Journal of Economics, vol. 82, pp. 465-473.

H. Low [1999] : Self-Insurance and Unemployment Benefit in a Life-Cycle Model of Labor Supply and Savings, Institute for Fiscal Studies, working paper $\mathrm{n}^{\circ} \mathrm{W} 99 / 24$.

R.E. Lucas [1987] : Models of Business Cycles, Yrjo Jahnsson Lectures Series, London : Blackwell.

C.F. Manski [1993] : Identification of Endogeneous Social Effects : the Reflection Problem, The Review of Economic Studies, vol. 60, pp. 531-542.

J.P. Martin [1996] : Indicateurs de taux de remplacement aux fins de comparaisons internationales, Revue économique de l'OCDE, vol. 26, pp. 115-132.

D. Montgomery [1991] : Social Networks and Labor Market Outcomes: Toward an Economic Analysis, American 
36 Yann Algan, Pierre Cahuc, Bruno Decreuse, François Fontaine, Solenne Tanguy

Economic Review, vol. 81, pp. 14081418.

R. Rogerson et T. Schindler [2002] : The Welfare Loss of Displaced Workers, Journal of Monetary Economics, vol. 49, pp. 1213-1234.

S. Shavell et L. Weiss [1979] : The Optimal Payment of Unemployment Insurance Benefits Over Time, Journal of Political Economy, vol. 87, pp. 13471362.

A. Stutzer et R. Lalive [2002] : The Role of Social Work Norms in Job Searching and Subjective Well-Being, Institute for Empirical Research in Economics, doc. de travail $\mathrm{n}^{\circ} 51$, université de Zurich.

G. Topa [2000] : Social Interactions, Local Spillovers and Unemployment, Review of Economic Studies, vol. 68, pp. 261-295.
G. Van den Berg et Van der Klaauw [2001] : Counseling and Monitoring of Unemployed Workers : Theory and Evidence from a Controlled Social Experiment, IZA discussion paper $n^{\circ} 374$.

C. Wang et S.D. Williamson [2002] : Moral Hazard, Optimal Unemployment Insurance and Experience Rating, Journal of Monetary Economics, vol. 49, pp. 1337-1372.

I. Werning [2002] : Optimal Unemployment Insurance with Unobservable Wealth, working paper, Chicago University.

J. Wilson [1987] : The Truly Disadvantaged: the Inner City, the Underclass and Public Policy, The University of Chicago Press. 